\title{
Self-Organized Critical Directed Percolation
}

\author{
Sergei Maslov ${ }^{(*, \dagger)}$ and Yi-Cheng Zhang ${ }^{(*)}$ \\ (*) Institut de Physique Théorique, Université de Fribourg, CH-1700, Switzerland \\ (†) Department of Physics, Brookhaven National Laboratory, Upton, New York 11973 \\ and Department of Physics, SUNY at Stony Brook, Stony Brook, New York 11974
}

(November 4, 2018)

\begin{abstract}
We introduce and study a dynamic transport model exhibiting Self-Organized Criticality. The novel concepts of our model are the probabilistic propagation of activity and unbiased random repartition of energy among the active site and its nearest neighbors. For space dimensionality $d \geq 2$ we argue that the model is related to $d+1$ dimensional directed percolation, with time interpreted as the preferred direction.
\end{abstract}

$05.40+\mathrm{j}, 64.60 \mathrm{Ht}, 64.60 \mathrm{Ak}, 05.70 \mathrm{Ln}$

Directed Percolation (DP) is one of the simplest and most recurrent models in statistical mechanics. Under very general guidelines (locality, scalar variable, etc.) Grassberger [1] and Janssen [2] have proposed that a wide range of models would fall into the DP universality class. This conjecture has stood the test of time. An impressive parade of models under various disguises turned out to belong to the same DP class (see [3] for a review). However, they all share one common feature: the activation probability $p$ has to be defined in advance, and when it is properly fine-tuned, a phase transition takes place. Our aim here is to design a dynamical model in which critical directed percolation would occur via self-organization.

Consider the following activation-transport problem. A conserved quantity called energy $E$ can be stored at each site of a $d$-dimensional square lattice with open boundaries. An input energy $\delta E<1$ is added to the fixed input site in the center of the system and this site is declared active. An active site can propagate activity under the following rules: 1) Random repartition of energy among the active site and its $2 d$ neighbors i.e. $E_{i} \rightarrow x_{i} \sum_{j=1}^{2 d+1} E_{j},(i=1, \ldots, 2 d+1)$, where $x_{i}=r_{i} / \sum_{j=1}^{2 d+1} r_{j}$, and $r_{i}$ are uncorrelated random numbers between 0 and $1 ; 2$ ) After repartition each of the above $2 d+1$ sites becomes active with the probability given by its energy content. If $E_{i}>1$ activation happens with certainty. There is no spontaneous activation of sites not connected to the current active site. The above process is repeated until no active sites are left in the system and then a new input is introduced. The above process strictly conserves energy except for the activation at the boundaries. Open boundaries allow the energy to escape.

It is easy to convince oneself that the system eventually organizes itself into a critical stationary state where the average stored energy $\bar{E}<1$. In this state the energy input and output through the boundaries balance each other, on average. The avalanches in this critical state have no characteristic size except the size of the system. The above model is inspired by the celebrated Bak-TangWiesenfeld (BTW) model of Self-Organized Criticality (SOC) 㘬. In fact it is a modified version of the SOC energy model introduced earlier [5]. The new feature here is that the activation probability is given by the transported energy.

In our model there exists an effective probability of activation of neighbors related to the average energy $\bar{E}$, stored in the system. Each of the $2 d+1$ sites participating in a single energy redistribution has equal chance to become active at the next time step. The propagation of activity in time is reminiscent of the model of epidemic

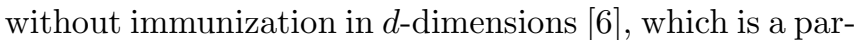
ticular realization of $d+1$-dimensional bond directed percolation with the preferred direction interpreted as time. The stationary state of our model corresponds to the critical disease propagation. In Fig. 11 we show a snapshot of the typical fractal pattern formed by active sites at a given time step in the two dimensional version of our model. Below we shall demonstrate that despite all the above suggestive considerations, the aim of mapping our model to Directed Percolation is only partially fulfilled: in $d \geq 2$ indeed the activation clusters seem to fall into the standard DP class; for the special case $d=1$ however, though still critical, our model belongs to a new class, probably corresponding to DP with long range correlated disorder.

There are three quantities characterizing an avalanche of activity: its spatial size $R$, its temporal duration $T$, and its volume, equal to the total number of activations $S$. They are connected via two scaling relations:

$$
\begin{aligned}
& S=R^{D} \\
& T=R^{z} .
\end{aligned}
$$

In the DP mapping these quantities are interpreted as the perpendicular and parallel size of cluster and its volume correspondingly. 
We have measured $S, T$ and $R$ for the avalanches in our model. The results of the numerical simulation in $1+1$ dimension are shown in Fig. 2. The exponent $D$ crosses over from $D=2.35 \pm 0.1$, which is close to the DP value $(2.32[7 \mid)$, to $1.3 \pm 0.1$. At the same time the exponent $z$ crosses over from $1.7 \pm 0.1$ to a much lower value of $1.0 \pm 0.1$. It is interesting to note that $S$ vs $T$ plot has a fairly good scaling of the form $S \sim T^{\alpha}$ throughout the whole region covered by our simulations. The exponent $\alpha=1.35 \pm 0.03$, is different from its DP value $\alpha=D / z=1.47[7]$. This suggests that in one dimension we are really dealing with a universality class different from 1+1-dimensional DP.

In order to check further the relation between our model and DP we have performed simulations in $2+1$ dimensions. The results are shown in Fig. 3. Here we have measured $D=2.92 \pm 0.05$ and $z=1.73 \pm 0.05$ in fair agreement with the DP values of 2.95 and 1.76 [8], respectively. No crossover was observed for system sizes up to $120 \times 120$. The scaling between $S$ and $T$ is the cleanest and the measured value of exponent $\alpha=1.675 \pm 0.01$ is in perfect agreement with its DP value of $\alpha=D / z=1.675 \pm 0.01$ [8]. We measured also the average stored energy $\bar{E}$ in critical state to be $0.393 \pm 0.005$, for $d=1$, and $0.210 \pm 0.005$, for $d=2$.

By definition, when the avalanche is over the energy at every site in the system is smaller than unity. But while it is running the energy is not bounded and in principle can diverge. We expect that the larger is the avalanche size $S$ the larger is the maximal energy $E_{\max }(S)$ reached during this avalanche. Our numerics indicate a very slow increase of $E_{\max }$ with $S$, for instance in $2+1$ dimensions $E_{\max }(S)$ increases from 1.18 to 1.75 while $S$ spans 6 decades. The observed increase in $E_{\max }(s)$ seems to be even slower than logarithmic, we were not able to determine whether it would saturate or diverge slowly. In any case we can say that empirically events $E_{i}>1$ are rare and the values are reasonably bounded. The overwhelming majority of the sites have simple probabilistic interpretation since $E_{i} \leq 1$.

Another difference between our model and DP is that while $p$ is a uniform constant for DP models, in our model $E_{i}$ is space dependent. If a particular site or a group of sites contain more energy than the average, this excess amount cannot immediately diffuse to infinity. The neighborhood in question will be more prone to activation, which may lead to spatial and temporal correlation in activity. Indeed this is the case for $d=1$. To detect spatial correlation in $E_{i}$, we have studied the fluctuations of the convolution of the energy variable: $\sigma(x)=\sum_{i=1}^{x}\left(E_{i}-\bar{E}\right)$. This method is known to be able to detect the presence of very weak correlations in $E_{i}$ [9]. For uncorrelated energies we would have $\sqrt{\left\langle\sigma(x)^{2}\right\rangle} \sim x^{0.5}$. Any deviation of the scaling exponent from 0.5 will indicate the presence of spatial correlation in $E_{i}$. In the one dimensional version of our model we have found this exponent to be in the range between 0.33 and 0.40 , quite far from the uncorrelated value 0.5 . This implies that $\sigma(x)$ performs so-called "persistent" random walk, being attracted to the regions it has visited before [10].

At first sight our model should be compared to the DP model with quenched disorder [11], where the probability of activation $p(i)$ is given for each site and never changes. In our model it is true that $E_{i}$ does not update when the activation is absent, thus in principle has a long memory. Activation reshuffles the energies in the neighborhood and $E_{i}$ becomes "annealed" as soon as it visits this site or its nearest neighbors. The conserved nature however implies that these are not completely fresh random numbers, as a genuine DP model would require. For the model to belong to DP universality class the excess of energy should be able to diffuse away fast enough. For $d=1$ as discussed above, this is not true and the spatial correlation of $E_{i}$ is relevant. The excellent agreement of the exponents of our model in 2 dimensions with 2+1-dimensional DP suggests that in high dimensions all these correlations are irrelevant. We conjecture that for $d \geq 2$ our model can effectively diffuse away any energy build-up and hence it belongs to the universality class of DP.

On a coarse-grained level the energy is very uniformly distributed. This homogeneity is remarkable especially taking into account that the energy input in our simulations is highly inhomogeneous: the energy is injected only at the central site. We have checked that the exponents do not change when the energy is added to a randomly selected site each time, much in the spirit of BTW model. This is another manifestation of the model's ability to organize itself to the unique critical state, independent of initial state or a particular type of driving.

In traditional SOC models activation occurs via a threshold mechanism. Though it is possible that in Nature threshold activation mechanisms play an important role, the probabilistic activation rules are surely more realistic: A site containing less energy than the threshold can still be activated by its active neighbors, but this occurs less likely. Our model can be called the probabilistic SOC. Probabilistic activation alone does not make any model to fall into the DP universality class. It is important that we use the neutral redistribution rule, when each of $2 d+1$ sites involved in redistribution gets an unbiased random share of the conserved energy. In traditional SOC models, like for instance in BTW-like models an active site always loses its energy to its neighbors. Therefore, during one avalanche its chances to be activated again are smaller than for its neighbors. We call such redistribution rules charitable. This leads to an effective repulsion of currently active sites from the previously activated region or outward bias in the spread of activity. Indeed, the dynamic exponent $z \sim 1.33$ in 2-dimensional BTW model with the charitable rule of 
dynamics is smaller than our $z=1.73$. That means that the outward expansion of activity is faster in BTW model due to repulsion. It is interesting to mention that simple balance of the velocity of moving active front to the total repulsion force acting on it gives the expression $z_{B T W}=\frac{d+2}{3}$ [5] which agrees very well with the results of numerical simulations of the BTW model. Finally a greedy redistribution rule where currently active sites on average gain energy can be envisaged. It is anticipated 12] that models with charitable, neutral and greedy rules should belong to different universality classes. The detailed analysis of how the above three different redistribution rules as well as threshold or probabilistic activation influence the universality class of SOC models will be relegated elsewhere.

In summary, we have introduced a simple probabilistic SOC model which is a modified version of the energy model [5]. The energy is introduced to the system at the central site, transported around through the conservative repartition of energy between currently active site and its nearest neighbors and dissipated at the boundary. The novel concepts of our model are:

1) A neutral rule of repartition of energy, where each site involved in it gets an unbiased random share of the strictly conserved sum.

2) The probability of the site to be activated by its active neighbor is given by its energy.

The system organizes itself to the critical state characterized by the power law distribution of avalanche sizes. The propagation of activity in time within the avalanche is conjectured to be equivalent to a model of spread of disease without immunization, which is a particular realization of bond directed percolation in $d+1$ dimension with preferred direction interpreted as time.

The work of one of us (SM) was supported by the U.S. Department of Energy Division of Material Science, under contract DE-AC02-76CH00016. SM thanks Institut de Phisique Théorique, Université de Fribourg for hospitality during the visit when this work was accomplished. We thank Per Bak for useful comments and careful reading of the manuscript.

[1] P. Grassberger, Z.Phys. B 47, 365 (1982).

[2] H. K. Janssen, Z. Phys. B 42, 151 (1981).

[3] P. Grassberger, J. Stat. Phys. 79, 13 (1995).

[4] P. Bak, C. Tang, and K. Wiesenfeld, Phys. Rev. Lett. 59, 381 (1987); and Phys. Rev. A 38, 364 (1988).

[5] Y.-C. Zhang, Phys. Rev. Lett. 63, 470 (1989).
[6] P. Grassberger, Math. Biosci. 62, 157 (1982); and J. Phys. A 17, L215 (1985).

[7] J.W. Essam, K. De'Bell, J. Adler and F. Bhatti, Phys. Rev. B 33, 1982 (1986).

[8] P. Grassberger, J. Phys. A 22, 3673 (1989).

[9] A. Schenkel, J. Zhang anf Y.-C. Zhang, Fractals 1, 41 (1993).

[10] B. B. Mandelbrot, The Fractal Geometry of Nature (W.H. Freeman, San Francisco, 1982).

[11] A. J. Noest, Phys. Rev. Lett. 57, 90 (1986).

[12] L. Pietronero, P. Tartaglia and Y.-C. Zhang, Physica A 173, 22 (1991); and Y.-C. Zhang, unpublished 1989. 


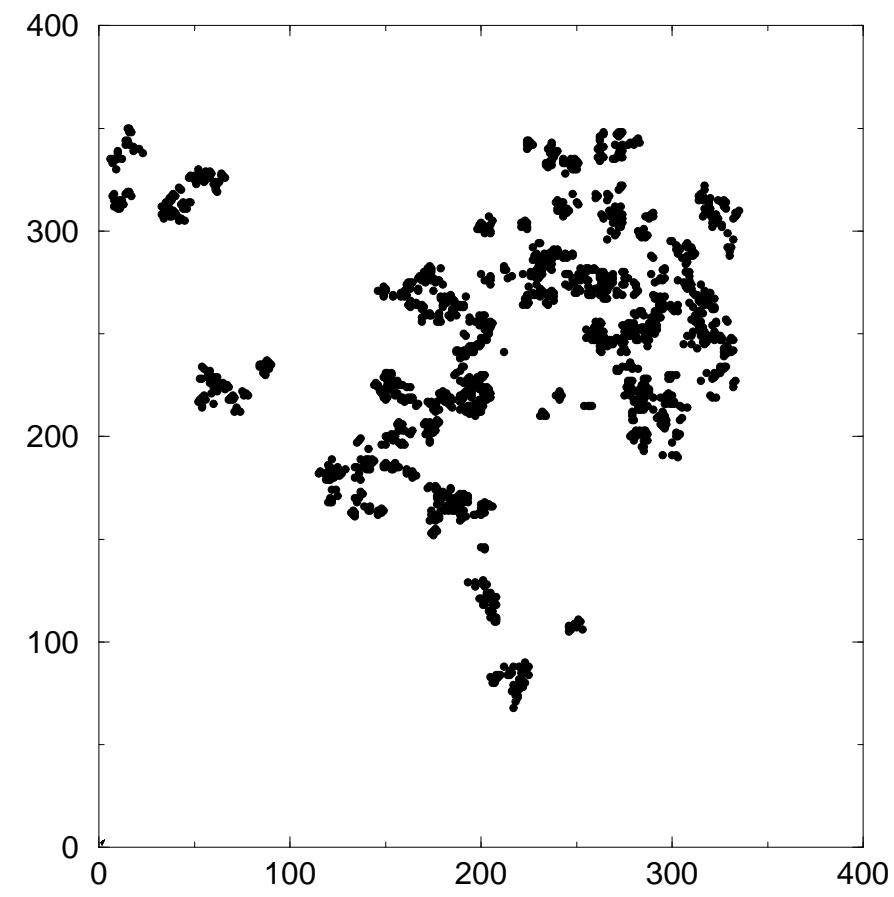

FIG. 1. Snapshot of activity in one realization of the avalanche process in the system of size $400 \times 400$. Active sites follow a fractal statistics

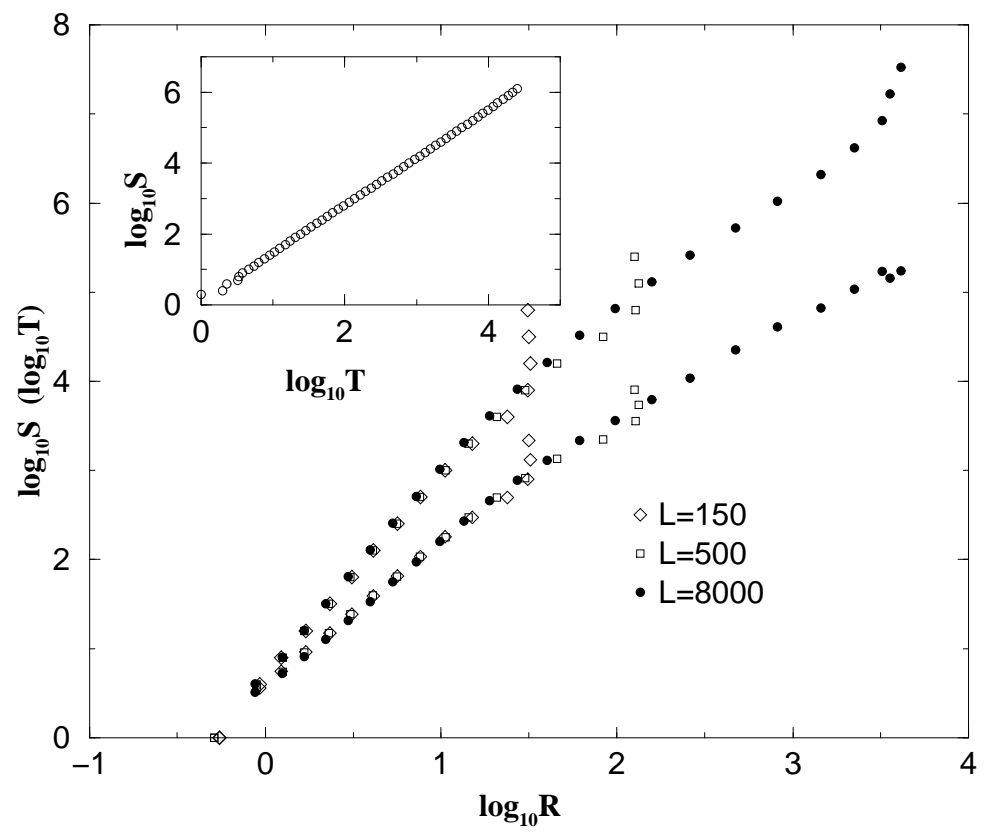

FIG. 2. The scaling of $S$ and $T$ with $R$ in one dimension for systems of different sizes. Crossover to smaller values of exponents $D$ and $z$ happens around $R=50$. The inset shows the $\log$ - $\log$ plot of $S$ vs $T$ in the system of size $L=3000$. There is no crossover observed and the scaling exponent $\alpha$ is measured to be $1.35 \pm 0.03$. 


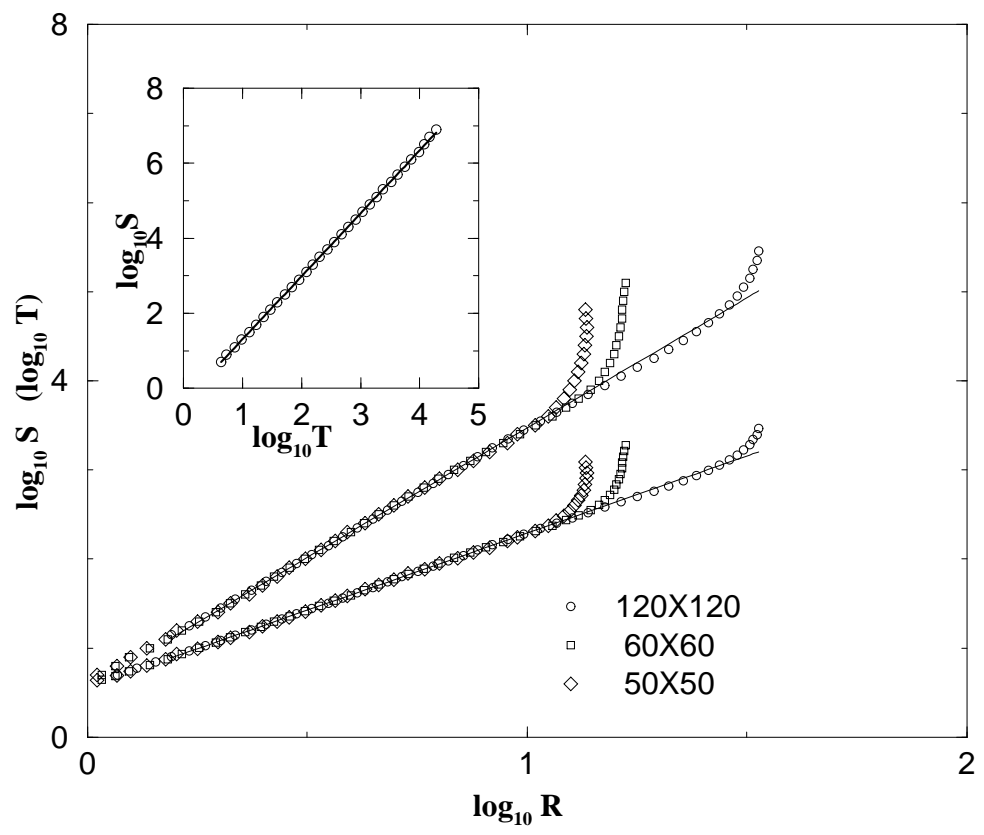

FIG. 3. The scaling of $S$ and $T$ with $R$ in two dimensions for systems of different sizes. Straight lines are fits, giving $D=2.92$ and $z=1.73$. The inset shows the scaling of $S$ with $T$. The measured exponent is $\alpha=1.675 \pm 0.010$. 